\title{
A note on topologically $b$-order bounded sets and generalized $b$-weakly compact operators
}

\author{
Nabil Machrafi ${ }^{1}$ (D), Birol Altin*2 (D) \\ ${ }^{1}$ Mohammed V University in Rabat, Faculty of Sciences, Department of Mathematics, Center CeReMAR, \\ B.P. 1014 RP, Rabat, Morocco \\ ${ }^{2}$ Department of Mathematics, Faculty of Science, Gazi University, 06500 Teknikokullar, Ankara
}

\begin{abstract}
We study the class of order weakly compact operators on locally solid Riesz spaces and the introduced class of generalized $b$-weakly compact operators on locally convex-solid Riesz spaces. We prove as a consequence that some well-known results on $b$-weakly compact operators on a Banach lattice extend in an improved version to generalized $b$-weakly compact operators on a Fréchet lattice.
\end{abstract}

Mathematics Subject Classification (2020). 46A40, 46B42, 47B60

Keywords. $b$-order bounded set, order weakly compact operator, $b$-weakly compact operator, locally convex-solid Riesz space, Fréchet lattice, Banach lattice

\section{Introduction and preliminaries}

In the Banach lattice framework, there is two interesting notions of weak compactness of operators: the order weak compactness and the $b$-weak compactness. Many interesting results on these two classes of operators have been established; see for instance [3, $5,6,11]$. In this paper, we investigate these two classes of operators in the more general frameworks of locally solid and locally convex-solid Riesz spaces. Such study, is motivated by the fact that these classes of operators have been extensively investigated in the literature in the restricted setting of Banach lattices, while the initial setting of their definitions does not require any topological structure (and a fortiori Banach lattice structure).

For locally convex-solid Riesz spaces, the order dual no longer coincide with the topological one, as is in the Banach lattice case. Consequently, new alternatives of the notions of $b$-order boundedness of sets and $b$-weak compactness of operators appear naturally, namely, the sets and operators that we call topologically $b$-order bounded and generalized $b$-weakly compact, respectively (Definitions $3.1,3.2$ ). We will give a complete answer on the relationship between the two notions of $b$-order boundedness and topological $b$-order boundedness of sets in Hausdorff locally convex-solid Riesz spaces (in particular, Fréchet lattices; Lemma 3.3 and Corollary 3.4). Also, we will be showing that the main Banach lattice results for order weakly compact and $b$-weakly compact operators extend naturally

\footnotetext{
*Corresponding Author.

Email addresses: nmachrafi@gmail.com,n.machrafi@um5r.ac.ma (N. Machrafi), birola@gazi.edu.tr (B. Altin)

Received: 02.07.2021; Accepted: 17.09.2021
} 
to our general frameworks for order weakly compact operators (Theorem 2.3) and generalized $b$-weakly compact operators (Theorems 3.11,3.18). Our strategy for the proofs consists on formulating and proving the equivalent versions of [3, Theorem 4.21] in our general settings (Lemmas 2.1,3.10), coming back to the Banach lattice setting through our lemmas, and using a very recent result of the authors [7] on a characterization of the class of $b$-weakly compact operators on a Banach lattice (Theorem 2.2).

We prove as a consequence that the term "Banach lattice" may be replaced by the term "Fréchet lattice" in an improved version for some main known results on $o$-weakly and $b$-weakly compact operators (Corollaries 2.7, 3.20), by adding some new sequential characterizations of these classes of operators.

Let us recall some concepts we will need in this paper. For a Hausdorff locally convex space $(N, \tau)$, the strong topology $\beta\left(N^{\prime}, N\right)$ on the topological dual $N^{\prime}$ of $N$ is the (locally convex) topology of uniform convergence on $\sigma\left(N, N^{\prime}\right)$-bounded subsets of $N$. The strong topology $\beta\left(N, N^{\prime}\right)$ on $N$ is defined similarly via $\sigma\left(N^{\prime}, N\right)$-bounded subsets of $N^{\prime}$. Throughout the paper, $N^{\prime \prime}$ will refer to the strong bidual of $N$, that is the topological dual of $\left(N^{\prime}, \beta\left(N^{\prime}, N\right)\right)$ endowed with the strong topology $\beta\left(N^{\prime \prime}, N^{\prime}\right)$.

A linear topology $\tau$ on a Riesz space $M$ (i.e. an ordered vector space $M$ such that any two vectors admit a supremum) is said to be locally solid, if it has a base of zero neighbourhoods consisting of solid sets. A subset $A \subset M$ is called solid if for every $x, y \in M,|x| \leq|y|$ and $y \in A$ imply $x \in A$, where $|x|:=\sup \{-x, x\}$. If the topology $\tau$ admits a base of zero neighbourhoods that are simultaneously convex and solid, then we call it locally convex-solid. A locally (convex-) solid Riesz space is a Riesz space endowed with a locally (convex-) solid topology. A Fréchet lattice is a completely metrizable locally convex-solid Riesz space. A Hausdorff locally convex-solid Riesz space is metrizable when its topology is induced by a translation-invariant distance, or equivalently, the topology is induced by a sequence of Riesz seminorms. A Banach lattice is a complete normed Riesz space (i.e., a Fréchet lattice whose topology is induced by a single Riesz norm). An example of a locally convex-solid Riesz space is the space $\mathbb{R}^{\Omega}$ of real-valued functions on a nonempty set $\Omega$, endowed with the pointwise ordering and the product topology. A typical example of a Fréchet lattice that is not a Banach lattice is the Riesz space $\mathbb{R}^{\mathbb{N}}$ of all real sequences endowed with the coordinatewise ordering and the product topology. A further example of a Fréchet lattice is the space $C(\Omega)$ of real-valued continuous functions on an open set $\Omega \subset \mathbb{R}^{N}$, endowed with the pointwise ordering and the topology of uniform convergence on compact subsets of $\Omega$.

Throughout the paper, the topological dual of a locally convex space $N$ and the order dual of a Riesz space $M$ will be denoted by $N^{\prime}$ and $M^{\sim}$, respectively. We mean by an operator between two vector spaces a linear mapping. For a continuous operator $H: N \rightarrow Y$ between two locally convex spaces, the adjoint (or the transpose) operator $H^{\prime}: Y^{\prime} \rightarrow N^{\prime}$ is defined by $H^{\prime}(f)=f \circ H, f \in Y^{\prime}$. This operator is continuous when $Y^{\prime}$ and $N^{\prime}$ are endowed with their strong topologies $\beta\left(Y^{\prime}, Y\right)$ and $\beta\left(N^{\prime}, N\right)$ (resp. weak* topologies $\sigma\left(Y^{\prime}, Y\right)$ and $\left.\sigma\left(N^{\prime}, N\right)\right)$. The second adjoint operator of $H$ is the adjoint operator of $H^{\prime}:\left(Y^{\prime}, \beta\left(Y^{\prime}, Y\right)\right) \rightarrow\left(N^{\prime}, \beta\left(N^{\prime}, N\right)\right)$, that is, the operator $H^{\prime \prime}: N^{\prime \prime} \rightarrow Y^{\prime \prime}$ such that $H^{\prime \prime}:=\left(H^{\prime}\right)^{\prime}$.

For more details and unexplained terminology and notations on locally solid Riesz spaces and Banach lattices, we refer the reader to [2-4].

\section{Order weakly compact operators on locally solid Riesz spaces}

Let $M$ be a Riesz space and $N$ be a Banach space. An operator $H: M \rightarrow N$ is said to be order bounded to bounded (or interval-bounded) if it maps any order bounded set in $M$ to a bounded set in $N$. In case $(M, \tau)$ is a Hausdorff locally solid Riesz space (in particular, a normed Riesz space), it is clear that a bounded (in particular, continuous) 
operator $H: M \rightarrow N$ is order bounded to bounded, for an order bounded set in $M$ is $\tau$-bounded. However, an order bounded to bounded operator $H: M \rightarrow N$ need not be bounded. On Fréchet lattices, order bounded to bounded operators and bounded (equivalently, continuous) operators are the same; see Corollary 3.9 hereafter.

The operator $H: M \rightarrow N$ is said to be order weakly (o-weakly) compact (or, of type A) if $H(A)$ is relatively weakly compact for every order bounded set $A \subset M$. Clearly, an $o$-weakly compact operator $H: M \rightarrow N$ is necessarily order bounded to bounded.

The following lemma can be proved by the similar idea in Theorem 4.21 in [3] and its proof is omitted.

Lemma 2.1. Let $(M, \tau)$ be a Hausdorff sequentially $\tau$-complete locally solid Riesz space. For $x \in M^{+}$, let $M_{x}$ denote the principal ideal generated by $x$. Then, the Riesz space $M_{x}$ is an AM-space under the norm

$$
\|u\|_{x}:=\inf \{\lambda>0:|u| \leq \lambda x\}, u \in M_{x} .
$$

Now, we state our first main result which gives several sequential characterizations of $o$-weakly compact operators on a locally solid Riesz space. The proof of these characterizations is mainly based on the above lemma and the result recently achieved by the authors [7] for $b$-weakly compact operators on Banach lattices, which reads as follows:

Theorem 2.2 ([7, Theorem 3.2]). Let $M, N$ be respectively a Banach lattice and a Banach space. Then, an operator $H: M \rightarrow N$ is b-weakly compact if and only if it is a weak KBoperator, i.e., for every increasing norm bounded sequence $\left(x_{n}\right) \subset M^{+},\left(H x_{n}\right)$ has a weakly convergent subsequence.

Theorem 2.3. Let $(M, \tau)$ be a Hausdorff sequentially $\tau$-complete locally solid Riesz space and $N$ be a Banach space. Then, for an order bounded to bounded operator $H: M \rightarrow N$, the following are equivalent:

(1) $H$ is an o-weakly compact operator.

(2) $\left\|H x_{n}\right\| \rightarrow 0$, for every disjoint order bounded sequence $\left(x_{n}\right) \subset M^{+}$.

(3) $\left(H x_{n}\right)$ is convergent for every increasing order bounded sequence $\left(x_{n}\right) \subset M^{+}$.

(4) $\left(H x_{n}\right)$ is weakly convergent for every increasing order bounded sequence $\left(x_{n}\right) \subset$ $M^{+}$.

(5) $\left(H x_{n}\right)$ has a convergent subsequence for every increasing order bounded sequence $\left(x_{n}\right) \subset M^{+}$.

(6) $\left(H x_{n}\right)$ has a weakly convergent subsequence for every increasing order bounded sequence $\left(x_{n}\right) \subset M^{+}$.

Proof. In all the proof, for $x \in M^{+}$we let $H_{x}$ denote the restriction of $H$ to $M_{x}$ endowed with $\|.\|_{x}$ and note that $H_{x}$ is clearly bounded (and hence continuous), for $H$ is an order bounded to bounded operator. Now, applying Lemma 2.1 together with [3, Theorem 5.52] and [11, Theorem 3.4.4] for a suitable $H_{x}, 1 \Leftrightarrow 2$ and $1 \Rightarrow 3$ follow. Note that $3 \Rightarrow 4 \Rightarrow 6$ and $3 \Rightarrow 5 \Rightarrow 6$ are obvious. So, what we need to prove here is $6 \Rightarrow 1$. Let $A$ be a set in $M$ with $A \subset[-x, x]$ for some $x \in M^{+}$. By Lemma 2.1, a norm bounded increasing sequence $\left(y_{n}\right) \subset\left(M_{x}\right)^{+}$is order bounded, and hence by the hypothesis $\left(H_{x} y_{n}\right)=\left(H y_{n}\right)$ has a weakly convergent subsequence. By Theorem $2.2, H_{x}$ is a $b$-weakly compact operator and hence $H(A)=H_{x}(A)$ is relatively weakly compact. This shows that $H$ is an $o$-weakly compact operator as desired.

Since for a Banach space $(N,\|\|$.$) the Mackey topology on N$ coincides with its norm topology $\tau_{\|.\|}$, one has

$$
\sigma\left(N, N^{\prime}\right) \subset \tau_{N} \subset \tau_{\|\cdot\|}
$$

for every locally convex topology $\tau_{N}$ on $N$ consistent with respect to the dual system $\left\langle N, N^{\prime}\right\rangle$; see [3, p 151]. Therefore, the following corollary follows immediately from the above theorem. 
Corollary 2.4. Under the same conditions of Theorem 2.3, an operator $H: M \rightarrow N$ is o-weakly compact iff $\left(H x_{n}\right)$ is $\tau_{N}$-convergent for every increasing order bounded sequence $\left(x_{n}\right) \subset M^{+}$and every locally convex topology $\tau_{N}$ on $N$ consistent with respect to the dual system $\left\langle N, N^{\prime}\right\rangle$.

In the setting of locally convex-solid Riesz spaces, we derive from the above theorem the following characterizations of continuous o-weakly compact operators.

Corollary 2.5. Let $(M, \tau)$ be a Hausdorff sequentially $\tau$-complete locally convex-solid Riesz space and $N$ be a Banach space. Then, for a continuous operator $H: M \rightarrow N$, the following are equivalent:

(1) $H$ is an o-weakly compact operator.

(2) $H^{\prime \prime}(I(M)) \subset N$, where $I(M)$ stands for the ideal generated by $M$ in $M^{\prime \prime}$.

(3) $H^{\prime \prime}\left(I(M)^{\uparrow}\right) \subset N$, where $I(M)^{\uparrow}:=\left\{x^{\prime \prime} \in I(M): \exists\left(x_{\alpha}\right) \subset M^{+}, x_{\alpha} \uparrow\left|x^{\prime \prime}\right|\right\}$.

Proof. Note that $1 \Leftrightarrow 2$ is given in [3, Exercise 22 p 339] without a proof. For sake of completeness we give a proof of it. For $1 \Rightarrow 2$, let $x^{\prime \prime} \in I(M)^{+}$and pick some $x \in M$ with $0 \leq x^{\prime \prime} \leq x$. By [3, Lemma 3.56] there exists a net $\left(x_{\alpha}\right) \subset[0, x] \subset M^{+}$with $x_{\alpha} \rightarrow x^{\prime \prime}$ for $\sigma\left(M^{\prime \prime}, M^{\prime}\right)$. Since $H^{\prime \prime}:\left(M^{\prime \prime}, \sigma\left(M^{\prime \prime}, M^{\prime}\right)\right) \rightarrow\left(N^{\prime \prime}, \sigma\left(N^{\prime \prime}, N^{\prime}\right)\right)$ is continuous, then $H x_{\alpha}=H^{\prime \prime} x_{\alpha} \rightarrow H^{\prime \prime} x^{\prime \prime}$ for $\sigma\left(N^{\prime \prime}, N^{\prime}\right)$. Now, by passing to a subnet, we may assume by the hypothesis that $H x_{\alpha} \rightarrow y$ for $\sigma\left(N, N^{\prime}\right)$ for some $y \in N$. From the uniqueness of the weak* limit in $N^{\prime \prime}$, we see that $H^{\prime \prime} x^{\prime \prime}=y$. This shows that $H^{\prime \prime}(I(M)) \subset N$ as desired.

$2 \Rightarrow 3$ is obvious.

$3 \Rightarrow 1$. Let $\left(x_{n}\right) \subset M$ be an increasing sequence with $0 \leq x_{n} \leq x$ for every $n$ and for some $x \in M^{+}$. Hence, $0 \leq x_{n} \uparrow x^{\prime \prime} \leq x$ in $M^{\prime \prime}$ for some $x^{\prime \prime} \in M_{+}^{\prime \prime}$ and we have that $H x_{n} \rightarrow H^{\prime \prime} x^{\prime \prime}$ for $\sigma\left(N^{\prime \prime}, N^{\prime}\right)$. Since $x^{\prime \prime} \in I(M)^{\uparrow}$, it follows by hypothesis that $H^{\prime \prime} x^{\prime \prime} \in N$ and therefore $H x_{n} \rightarrow H^{\prime \prime} x^{\prime \prime}$ for $\sigma\left(N, N^{\prime}\right)$. This shows by Theorem 2.3 (4) that $H$ is an $o$-weakly compact operator.

Remark 2.6. Compared to [3, Theorem 5.57] and [11, Theorem 3.4.4], Theorem 2.3 (4) - (6) and Corollary 2.5 (3) give new characterizations for the class of $o$-weakly compact operators. The other characterizations of Theorem 2.3 and Corollary 2.5 are due to P. G. Dodds [8] in the Banach lattice case.

As a further consequence, we get an improvement of [11, Theorem 3.4.4] and [3, Theorem 5.57], for o-weakly compact operators on Fréchet lattices (in particular, Banach lattices).

Corollary 2.7. Let $M, N$ be a Fréchet lattice and a Banach space, respectively. Then, for a continuous operator $H: M \rightarrow N$, the following are equivalent:

(1) $H$ is an o-weakly compact operator.

(2) $\left\|H x_{n}\right\| \rightarrow 0$, for every disjoint order bounded sequence $\left(x_{n}\right) \subset M^{+}$.

(3) $\left(H x_{n}\right)$ is convergent for every increasing order bounded sequence $\left(x_{n}\right) \subset M^{+}$.

(4) $\left(H x_{n}\right)$ is weakly convergent for every increasing order bounded sequence $\left(x_{n}\right) \subset$ $M^{+}$.

(5) $\left(H x_{n}\right)$ has a convergent subsequence for every increasing order bounded sequence $\left(x_{n}\right) \subset M^{+}$.

(6) $\left(H x_{n}\right)$ has a weakly convergent subsequence for every increasing order bounded sequence $\left(x_{n}\right) \subset M^{+}$.

(7) $H^{\prime \prime}(I(M)) \subset N$.

(8) $H^{\prime \prime}\left(I(M)^{\uparrow}\right) \subset N$.

\section{Generalized $b$-weakly compact operators on locally convex-solid Riesz spaces}

To state the bidual versions of the above results, we need to recall some concepts. A Riesz space $M$, with a separating order dual, embeds naturally in its order bidual 
$M^{\sim \sim}:=\left(M^{\sim}\right)^{\sim}$ via the lattice embedding $x \rightarrow \tilde{x}$ of $M$ into $M^{\sim \sim}$ defined by

$$
\tilde{x}(f)=f(x), f \in M^{\sim} .
$$

Therefore, from [5] a subset $A \subset M$ is said to be $b$-order bounded if it is order bounded as a subset of $M^{\sim \sim}$, that is, there exists $0 \leq \varphi \in M^{\sim \sim}$ with $A \subset[-\varphi, \varphi]$ in $M^{\sim \sim}$.

A typical case in which the Riesz space $M$ has a separating order dual is when $M$ is a Hausdorff locally convex-solid Riesz space, since in this case $M^{\prime} \subset M^{\sim}$; see [3, Theorem 3.49]. In such case, another type of the notion of $b$-order boundedness of sets appears naturally. Indeed, the strong dual $\left(M^{\prime}, \beta\left(M^{\prime}, M\right)\right)$ is also a Hausdorff locally convex-solid Riesz space and the natural embedding $x \rightarrow \widehat{x}$ of $M$ into its strong bidual $M^{\prime \prime}$ defined by

$$
\widehat{x}\left(x^{\prime}\right)=x^{\prime}(x), x^{\prime} \in M^{\prime}
$$

is a Riesz isomorphism. Under this embedding, we will identify in what follows $M$ with a Riesz subspace of $M^{\prime \prime}$. For the details of the above facts, see [2, p 80-81].

So, we are now in position to introduce the new concepts in relation with a new type of $b$-order boundedness of sets in Hausdorff locally convex-solid Riesz spaces.

Definition 3.1. Let $M$ be a Hausdorff locally convex-solid Riesz space and $N$ be a Banach space.

(1) A subset $A \subset M$ is said to be topologically $b$-order bounded if it is order bounded as a subset of $M^{\prime \prime}$, that is there exists $x^{\prime \prime} \in M_{+}^{\prime \prime}$ with $A \subset\left[-x^{\prime \prime}, x^{\prime \prime}\right]$ in $M^{\prime \prime}$.

(2) An operator $H: M \rightarrow N$ is said to be topologically $b$-order bounded to bounded if it maps any topologically $b$-order bounded set in $M$ to a bounded set in $N$.

Let $M, N$ be a Banach lattice and a Banach space, respectively. From [5], an operator $H: M \rightarrow N$ is said to be $b$-weakly compact (or of type B), if $H$ maps $b$-order bounded sets in $M$ to relatively weakly compact sets in $N$. We introduce in the following definition a generalized concept for this class of operators in the framework of Hausdorff locally convex-solid Riesz spaces.

Definition 3.2. Let $M$ be a Hausdorff locally convex-solid Riesz space and $N$ be a Banach space. An operator $H: M \rightarrow N$ is said to be generalized $b$-weakly compact (or briefly, gbwc ), if $H(A)$ is relatively weakly compact for every topologically $b$-order bounded set $A \subset M$.

In case $M$ is a Banach lattice, the strong topology $\beta\left(M^{\prime}, M\right)$ coincides with the usual norm topology on $M^{\prime}$, and hence the strong bidual $M^{\prime \prime}$ coincides with the usual norm bidual of $M$, which in turn coincides with the order bidual $M^{\sim \sim}$ of $M$. It follows that in this case, the notion of topological $b$-order boundedness of sets (resp. $g b$-weak compactness of operators) is nothing but that of $b$-order boundedness (resp. $b$-weak compactness).

The following result gives us useful characterizations of the above two types of $b$-order boundedness in Hausdorff locally convex-solid Riesz spaces, and consequently shows us that these two notions still coincide in the setting of Fréchet lattices.

Theorem 3.3. Let $M$ be a Hausdorff locally convex-solid Riesz space. For a subset $A \subset$ $M$, let $F_{A}\left(r e s p . S_{A}\right)$ be the set of infima (resp. suprema) of finitely many elements of $A$. Then,

(1) $A$ is topologically b-order bounded iff $-\infty<\inf f\left(F_{A}\right) \leq \sup f\left(S_{A}\right)<+\infty$ for every $f \in M_{+}^{\prime}$;

(2) $A$ is b-order bounded iff $-\infty<\inf f\left(F_{A}\right) \leq \sup f\left(S_{A}\right)<+\infty$ for every $f \in M_{+}^{\sim}$.

Proof. 1. For the only if part, let $x^{\prime \prime}, y^{\prime \prime} \in M^{\prime \prime}$ with $A \subset\left[y^{\prime \prime}, x^{\prime \prime}\right]$ in $M^{\prime \prime}$. For every $f \in M_{+}^{\prime}$, every $x \in F_{A}$ and every $y \in S_{A}$, we have $y^{\prime \prime}(f) \leq f(x)$ and $f(y) \leq x^{\prime \prime}(f)$. Therefore,

$$
y^{\prime \prime}(f) \leq \inf f\left(F_{A}\right) \leq \sup f\left(S_{A}\right) \leq x^{\prime \prime}(f)
$$


for every $f \in M_{+}^{\prime}$, as desired.

For the if part, since the topological $b$-order boundedness of $A$ and the hypothesis are preserved by passing to a translation of $A$ by an element of $M$, we may assume without loss of generality that $0 \in A$. Let $\varphi: M_{+}^{\prime} \rightarrow \mathbb{R}^{+}$be the mapping defined by $\varphi(f)=\sup f\left(S_{A}\right)$. Since $S_{A}$ is an upward directed set, the mapping $\varphi$ is additive. Indeed, let $f, g \in M_{+}^{\prime}$. For $x \in S_{A}$ we have

$$
(f+g)(x)=f(x)+g(x) \leq \varphi(f)+\varphi(g) .
$$

Therefore, $\varphi(f+g) \leq \varphi(f)+\varphi(g)$. Now, for $x, y \in S_{A}$ pick some $z \in S_{A}$ with $x \leq z$ and $y \leq z$. Hence,

$$
f(x)+g(y) \leq f(z)+g(z)=(f+g)(z) \leq \varphi(f+g) .
$$

It follows that $\varphi(f)+\varphi(g) \leq \varphi(f+g)$, and hence the equality. By Kantorovich extension theorem $\varphi$ extends uniquely to an element of $\left(M^{\prime}\right)_{+}^{\sim}$, denoted again by $\varphi$, and we have that $\sup S_{A}=\varphi$ in $M^{\prime \prime}$. Indeed, we shall first show that $\varphi \in M^{\prime \prime}$, that is $\varphi$ is continuous. To this end, it suffices to show that the restriction of $\varphi$ to $M_{+}^{\prime}$ is continuous at zero, since $\left(M^{\prime}, \beta\left(M^{\prime}, M\right)\right)$ is a Hausdorff locally convex-solid Riesz space. Let $\left(f_{\alpha}\right)$ be a net in $M_{+}^{\prime}$ with $f_{\alpha} \rightarrow 0$ for $\beta\left(M^{\prime}, M\right)$. That is $\left(f_{\alpha}\right)$ converges uniformly to zero on every $\sigma\left(M, M^{\prime}\right)$-bounded set in $M$. Or,

$$
0 \leq \varphi\left(f_{\alpha}\right)=\sup f_{\alpha}\left(S_{A}\right) \leq \sup _{x \in S_{A}}\left|f_{\alpha}(x)\right| \rightarrow 0,
$$

for $S_{A}$ is by hypothesis a $\sigma\left(M, M^{\prime}\right)$-bounded set in $M$. This shows that $\varphi\left(f_{\alpha}\right) \rightarrow 0$ and then $\varphi \in\left(M^{\prime}, \beta\left(M^{\prime}, M\right)\right)^{\prime}=M^{\prime \prime}$ as desired. Now, for $x \in S_{A}, f(x) \leq \varphi(f)$ for every $f \in M_{+}^{\prime}$, that is $x \leq \varphi$ in $M^{\prime \prime}$ for every $x \in S_{A}$. Let $\psi \in M^{\prime \prime}$ with $x \leq \psi$ in $M^{\prime \prime}$ for every $x \in S_{A}$. For $f \in M_{+}^{\prime}$, we have $f(x) \leq \psi(f)$ for every $x \in S_{A}$. Therefore, $\varphi(f) \leq \psi(f)$ for every $f \in M_{+}^{\prime}$, that is $\varphi \leq \psi$ in $M^{\prime \prime}$ and then the desired conclusion.

Now, from $\sup f\left(S_{-A}\right)=-\inf f\left(F_{A}\right)<+\infty$, it follows similarly the existence of $\psi \in M^{\prime \prime}$ with inf $F_{A}=-\sup S_{-A}=\psi$ in $M^{\prime \prime}$. This shows that $A \subset F_{A} \cap S_{A} \subset[\psi, \varphi]$ in $M^{\prime \prime}$, that is $A$ is topologically $b$-order bounded as desired.

2. The proof is similar to 1 and therefore is omitted.

Corollary 3.4. Let $M$ be a Hausdorff locally convex-solid Riesz space. Then, every border bounded set in $M$ is topologically b-order bounded. If furthermore $M$ is a Fréchet lattice, then a set in $M$ is b-order bounded iff it is topologically b-order bounded.

Proof. This follows from the inclusion $M^{\prime} \subset M^{\sim}$ with equality holds if $M$ is a Fréchet lattice; see [1, Theorem 9.11].

In Hausdorff locally convex-solid Riesz spaces, the two notions of $b$-order boundedness and topological $b$-order boundedness of sets are different, as the following example shows.

Example 3.5. Let $M=c_{00}$ be the Riesz space of eventually zero real sequences endowed with the coordinatewise ordering and the Riesz norm defined by $\left\|\left(x_{n}\right)\right\|_{\infty}=\sup _{n}\left|x_{n}\right|$. Thus, $\left(c_{00}\right)^{\prime \prime}=l_{\infty}$ and $\left(c_{00}\right)^{\sim \sim}=c_{00}$. Hence, if for $n \in \mathbb{N} u_{n}=\sum_{k=1}^{n} e_{k}$, where $e_{n}$ denotes the unit vector in $c_{00}$ with one in the $n$th coordinate and zero elsewhere, the set $\left\{u_{n}: n \in \mathbb{N}\right\} \subset c_{00}$ is topologically $b$-order bounded which fails to be $b$-order bounded.

Next, we point out that the coincidence of topologically $b$-order bounded sets and $b$ order bounded sets does not characterize Fréchet lattices, as the following two examples show. The second example shows that the topological bidual and the order bidual of a Hausdorff locally convex-solid Riesz space may coincide even in the non-Banach lattice setting.

Example 3.6. Let $M=l_{\infty}$ be endowed with the coordinatewise ordering and the absolute weak* topology $|\sigma|\left({ }_{\infty}, l_{1}\right)$. Then, [3, Theorem 3.57] combined with [3, Theorem 4.9] show that $M^{\prime \prime}=M$. Thus, topologically $b$-order bounded sets and order bounded sets of $M$ are 
the same. Now, since $M$ is an order dual, it has the property (b), that is, $b$-order bounded sets and order bounded sets of $M$ are the same; see [5, Example 1.2].

Example 3.7. Let $M=c_{0}$ be endowed with the coordinatewise ordering and the absolute weak topology $|\sigma|\left(c_{0}, l_{1}\right)$. Then, [3, Theorem 3.50] shows that $M^{\prime \prime}=l_{\infty}$ and we have that $M^{\sim \sim}=l_{\infty}$. This shows that topologically $b$-order bounded sets and $b$-order bounded sets of $M$ are the same.

The following proposition shows that topologically $b$-order bounded sets in a Hausdorff locally convex-solid Riesz space are topologically bounded.

Proposition 3.8. Every topologically b-order bounded set in a Hausdorff locally convexsolid Riesz space $(M, \tau)$ is $\tau$-bounded.

Proof. Let $A \subset M$ be a topologically $b$-order bounded set. Since the absolute weak* topology $|\sigma|\left(M^{\prime \prime}, M^{\prime}\right)$ is Hausdorff locally convex-solid on $M^{\prime \prime}, A$ is $|\sigma|\left(M^{\prime \prime}, M^{\prime}\right)$-bounded. Since $|\sigma|\left(M^{\prime \prime}, M^{\prime}\right)$ induces on $M$ the absolute weak topology $|\sigma|\left(M, M^{\prime}\right)$, then $A$ is $|\sigma|\left(M, M^{\prime}\right)$-bounded. Now, we know that the $|\sigma|\left(M, M^{\prime}\right)$-bounded sets and the $\tau$-bounded sets of $M$ are the same, for $|\sigma|\left(M, M^{\prime}\right)$ is a consistent topology with respect to the Riesz dual system $\left\langle M, M^{\prime}\right\rangle$; see [3, Theorems 3.25, 3.50]. This completes the proof.

We note, clearly, that a $g b$-weakly compact operator $H: M \rightarrow N$, from a Hausdorff locally convex-solid Riesz space $M$ to a Banach space $N$, is necessarily topologically $b$ order bounded to bounded, and that the class of $g b$-weakly compact operators from $M$ to $N$ is contained in that of $o$-weakly compact operators from $M$ to $N$, and contains, by the above proposition, that of weakly compact operators from $M$ to $N$ (i.e. operators that maps bounded sets of $M$ to relatively weakly compact ones of $N$ ). Note that the latter inclusions are strict in general, as it is already the case when $M$ is a Banach lattice.

The following corollary deals with the relationship between the other classes of operators involving the above types of boundedness.

Corollary 3.9. For an operator $H: M \rightarrow N$ from a Hausdorff locally convex-solid Riesz space to a Banach space, consider the following statements:

(1) $H$ is continuous.

(2) $H$ is bounded.

(3) $H$ is topologically b-order bounded to bounded.

(4) $H$ is order bounded to bounded.

Then, $1 \Rightarrow 2 \Rightarrow 3 \Rightarrow 4$. If, furthermore, $M$ is a Fréchet lattice, then the above statements are equivalent.

Proof. $2 \Rightarrow 3$ follows from Proposition 3.8 and $3 \Rightarrow 4$ is obvious. In case $M$ is a Fréchet lattice, $4 \Rightarrow 1$ is by $[9$, Theorem 1.3$]$.

Let us recall that a Hausdorff locally convex space $(N, \tau)$ is said to be barrelled if the barrels form a base of $\tau$-neighbourhoods of zero. A barrel of $N$ is a closed absorbent absolutely convex set. A characterization of a Hausdorff locally convex space $(N, \tau)$ to be barrelled is that the strong topology $\beta\left(N, N^{\prime}\right)$ coincide with the original topology $\tau$ of $N$; see [10, p 257].

A subset $A$ of a vector space absorbs a set $B$ if $\lambda B \subset A$ for some $\lambda>0$. A Hausdorff locally convex space $(N, \tau)$ is said to be quasi-barrelled if every bornivorous barrel in $N$ is a $\tau$-neighbourhood of zero. Here, we mean by a bornivorous barrel a barrel that absorbs all the bounded sets of $N$. It is so clear that a barrelled space is quasi-barrelled. Metrizable locally convex spaces (in particular, normed spaces) are examples of quasibarrelled spaces, while complete metrizable locally convex spaces (in particular, Banach spaces) are examples of barrelled spaces. 
Now, we state the bidual version of Lemma 2.1 which will be used mainly in the proof of the first main result of this section.

Lemma 3.10. Let $(M, \tau)$ be a Hausdorff sequentially $\tau$-complete and quasi-barrelled locally convex-solid Riesz space. For $x^{\prime \prime} \in M_{+}^{\prime \prime}$, let $Y_{x^{\prime \prime}}:=M_{x^{\prime \prime}} \cap M$, where $M_{x^{\prime \prime}}$ is the principal ideal generated by $x^{\prime \prime}$ in $M^{\prime \prime}$. Then, the Riesz space $Y_{x^{\prime \prime}}$ is an AM-space under the norm

$$
\|u\|_{x^{\prime \prime}}=\inf \left\{\lambda>0:|u| \leq \lambda x^{\prime \prime}\right\}, u \in Y_{x^{\prime \prime}} .
$$

Proof. It is not difficult to show that $\|\cdot\|_{x^{\prime \prime}}$ defines a lattice norm on $Y_{x^{\prime \prime}}$ satisfying $\|u \vee v\|_{x^{\prime \prime}}=\max \left(\|u\|_{x^{\prime \prime}},\|v\|_{x^{\prime \prime}}\right)$ whenever $u \wedge v=0$. So, only we need to prove that $Y_{x^{\prime \prime}}$ is norm complete. Assume that a sequence $\left(x_{n}\right)$ of $Y_{x^{\prime \prime}}$ is norm Cauchy. Since $\left(M^{\prime \prime}, \beta\left(M^{\prime \prime}, M^{\prime}\right)\right)$ is a Hausdorff locally convex-solid Riesz space, by the same consideration of the proof of Theorem 4.21 [3] we see that there exists a subsequence $\left(x_{n_{k}}\right)$ of $\left(x_{n}\right)$ with

$$
\left|x_{n_{k}+p}-x_{n_{k}}\right| \leq 2^{-k} x^{\prime \prime} \forall k, p \in \mathbb{N},
$$

and that $\left(x_{n_{k}}\right)$ is $\beta\left(M^{\prime \prime}, M^{\prime}\right)$-Cauchy in $M^{\prime \prime}$. Since $M$ is sequentially $\tau$-complete and quasi-barrelled, it is barrelled; see [10, p 368]. It follows that the topology $\beta\left(M^{\prime \prime}, M^{\prime}\right)$ induces $\beta\left(M, M^{\prime}\right)=\tau$ on $M$; see [10, p 300-301]. Therefore, $\left(x_{n_{k}}\right)$ is $\tau$-Cauchy. Now, the rest of the proof is similar to that of the proof of Theorem 4.21 [3].

Next, we state our following main result which gives several sequential characterizations of $b$-weakly compact operators on a locally convex-solid Riesz space. The proof of these characterizations is mainly based on the above lemma and Theorem 2.2.

Theorem 3.11. Let $(M, \tau)$ be a Hausdorff sequentially $\tau$-complete and quasi-barrelled locally convex-solid Riesz space and $N$ be a Banach space. Then, for a topologically $b$ order bounded to bounded operator $H: M \rightarrow N$, the following are equivalent:

(1) $H$ is a gbwc operator.

(2) $\left\|H x_{n}\right\| \rightarrow 0$, for every disjoint topologically b-order bounded sequence $\left(x_{n}\right) \subset M^{+}$.

(3) $\left(H x_{n}\right)$ is convergent for every increasing topologically b-order bounded sequence $\left(x_{n}\right) \subset M^{+}$.

(4) $\left(H x_{n}\right)$ is weakly convergent for every increasing topologically b-order bounded sequence $\left(x_{n}\right) \subset M^{+}$.

(5) $\left(H x_{n}\right)$ has a convergent subsequence for every increasing topologically b-order bounded sequence $\left(x_{n}\right) \subset M^{+}$.

(6) $\left(H x_{n}\right)$ has a weakly convergent subsequence for every increasing topologically border bounded sequence $\left(x_{n}\right) \subset M^{+}$.

Proof. In all the proof, for $x^{\prime \prime} \in M_{+}^{\prime \prime}$ we let $H_{x^{\prime \prime}}$ denote the restriction of $H$ to $Y_{x^{\prime \prime}}$ endowed with $\|\cdot\|_{x^{\prime \prime}}$ and note that $H_{x^{\prime \prime}}$ is clearly bounded (and hence continuous), for $H$ is a topologically $b$-order bounded to bounded operator. Now, applying Lemma 3.10 for a suitable $H_{x^{\prime \prime}}, 1 \Leftrightarrow 2$ and $1 \Rightarrow 3$ follow by the same arguments as in [5, Proposition 2.8] and [6, Proposition 1]. Note that $3 \Rightarrow 4 \Rightarrow 6$ and $3 \Rightarrow 5 \Rightarrow 6$ are obvious. So, what we need to prove here is $6 \Rightarrow 1$. Let $A$ be a set in $M$ with $A \subset\left[-x^{\prime \prime}, x^{\prime \prime}\right]$ in $M^{\prime \prime}$ for some $x^{\prime \prime} \in M_{+}^{\prime \prime}$. By Lemma 3.10, a norm bounded increasing sequence $\left(y_{n}\right) \subset\left(Y_{x^{\prime \prime}}\right)^{+}$is topologically $b$ order bounded, and hence by the hypothesis $\left(H_{x^{\prime \prime}} y_{n}\right)=\left(H y_{n}\right)$ has a weakly convergent subsequence. It follows from Theorem 2.2 that $H_{x^{\prime \prime}}$ is a $b$-weakly compact operator. Now, since $Y_{x^{\prime \prime}}$ is an AM-space, $Y_{x^{\prime \prime}}^{\prime \prime}$ is an AM-space with a unit $e$ such that its closed unit ball $B_{Y_{x^{\prime \prime}}^{\prime \prime}}=[-e, e]$. Since $Y_{x^{\prime \prime}}$ is isometrically lattice embedded in $Y_{x^{\prime \prime}}^{\prime \prime}$, then the closed unit ball $B_{Y_{x^{\prime \prime}}}=Y_{x^{\prime \prime}} \cap[-e, e]$. Now, from $A \subset B_{Y_{x^{\prime \prime}}} \subset[-e, e]$ we see that $A$ is $b$-order bounded as a subset of the AM-space $Y_{x^{\prime \prime}}$. Therefore, $H(A)=H_{x^{\prime \prime}}(A)$ is relatively weakly compact. This shows that $H$ is a gbwc operator as desired. 
Similarly as for Corollary 2.4, we have the following one.

Corollary 3.12. Under the same conditions of Theorem 3.11, an operator $H: M \rightarrow N$ is gb-weakly compact iff $\left(H x_{n}\right)$ is $\tau_{N}$-convergent for every increasing topologically b-order bounded sequence $\left(x_{n}\right) \subset M^{+}$and every locally convex topology $\tau_{N}$ on $N$ consistent with respect to the dual system $\left\langle N, N^{\prime}\right\rangle$.

By the same arguments of the proof of Corollary 2.5 we derive from Theorem 3.11 (4) the following characterization of continuous $g b$-weakly compact operators.

Corollary 3.13. Let $(M, \tau)$ be a Hausdorff sequentially $\tau$-complete and quasi-barrelled locally convex-solid Riesz space and $N$ be a Banach space. Then, a continuous operator $H: M \rightarrow N$ is gb-weakly compact iff $H^{\prime \prime}\left(M^{\uparrow}\right) \subset N$, where $M^{\uparrow}:=\left\{x^{\prime \prime} \in M^{\prime \prime}: \exists\left(x_{\alpha}\right) \subset\right.$ $\left.M^{+}, x_{\alpha} \uparrow\left|x^{\prime \prime}\right|\right\}$.

Remark 3.14. Compared to [5, Propositions 2.8, 2.11] and [6, Proposition 1], Theorem 3.11 (4) - (6) and Corollary 3.13 give new characterizations for the class of $b$-weakly compact operators. The other characterizations of Theorems 3.11, 3.18 are due to S. Alpay, B. Altin, C. Tonyali [5] and B. Altin [6], in the Banach lattice case.

The well-known domination property for $b$-weakly compact operators [5, Corollary 2.9] extends to the class of $g b$-weakly compact operators. We formulate it, in the following corollary, in a more general setting than that when the range space of operators is a Banach lattice. For that, let us recall that an ordered Banach space $N$ is a real Banach space endowed with a partial order relation induced by a closed cone $N^{+}$in $N$. The cone $N^{+}$is said to be normal, if $N$ admits an equivalent monotone norm $\|$.$\| , i.e., an equivalent$ norm $\|$.$\| such that for every x, y \in N$,

$$
0 \leq x \leq y \Rightarrow\|x\| \leq\|y\| .
$$

Note that the vector space of all operators from an ordered vector space $M$ with a generating cone to an ordered vector space $N$ is ordered via the cone of positive operators, i.e., operators $H: M \rightarrow N$ such that $H\left(M^{+}\right) \subset N^{+}$. Now, we point out that a positive operator $H: M \rightarrow N$, from a Hausdorff locally convex-solid Riesz space to an ordered Banach space with a normal cone, need not be topologically $b$-order bounded to bounded, as shown in the following example, which is by Corollary 3.9 an extension of [1, Example 9.7].

Example 3.15. Let $f: c_{00} \rightarrow \mathbb{R}$ be the linear functional defined by

$$
f\left(\left(x_{n}\right)\right)=\sum_{n=1}^{\infty} x_{n}
$$

Then, clearly, $f$ is positive, but if for $n \in \mathbb{N} u_{n} \in c_{00}$ is the vector defined as in Example 3.5 , then the set $\left\{u_{n}: n \in \mathbb{N}\right\}$ is topologically b-order bounded, but $\left\{f\left(u_{n}\right)=n: n \in \mathbb{N}\right\}$ is unbounded in $\mathbb{R}$.

The following corollary follows now from Theorem 3.11 (2), since the ordered Banach space $N$ admits an equivalent monotone norm.

Corollary 3.16. Let $(M, \tau)$ be a Hausdorff sequentially $\tau$-complete and quasi-barrelled locally convex-solid Riesz space and $N$ be an ordered Banach space with a normal cone. Let $H, S: M \rightarrow N$ be two topologically b-order bounded to bounded operators such that $0 \leq H \leq S$. Then, $H$ is a gb-weakly compact operator whenever $S$ is one.

To state the equivalent of Corollary 2.5 (2) for $g b$-weakly compact operators, we need to recall the following concepts: a Hausdorff locally solid Riesz space $(M, \tau)$ is said to have the Lebesgue (resp. $\sigma$-Lebesgue) property (or the topology $\tau$ is order continuous (resp. $\sigma$-order continuous)) if for every net $\left(x_{\alpha}\right) \subset M$ (resp. sequence $\left.\left(x_{n}\right) \subset M\right), x_{\alpha} \downarrow 0$ (resp. 
$x_{n} \downarrow 0$ ) implies that $\left(x_{\alpha}\right) \tau$-converges to 0 (resp. $\left(x_{n}\right) \tau$-converges to 0 ). Note that, in Fréchet lattices the Lebesgue property is equivalent to the $\sigma$-Lebesgue property and the $(\sigma-)$ Dedekind completeness.

We will use the following lemma whose proof follows directly from [2, Theorem 3.13] combined with [3, Theorem 3.57].

Lemma 3.17. If $M$ is a Dedekind complete Hausdorff locally convex-solid Riesz space with the Lebesgue property, then $M$ is an ideal in $M^{\prime \prime}$.

Note that under the conditions of the above lemma $M^{\uparrow}=B(M)$, the band generated by $M$ in $M^{\prime \prime}$; see [3, Theorem 1.38]. The result in Corollary 3.13 can in this case be improved as follows:

Theorem 3.18. Let $(M, \tau)$ be a Dedekind complete Hausdorff locally convex-solid Riesz space with the Lebesgue property and $N$ be a Banach space. Then, a continuous operator $H: M \rightarrow N$ is gb-weakly compact iff $H^{\prime \prime}(B(M)) \subset N$.

Proof. Assume that $H$ is $g b$-weakly compact. Since by Lemma $3.17 M$ is an ideal in $M^{\prime \prime}$, it follows that $M^{\uparrow}=B(M)$. Therefore, for $x^{\prime \prime} \in B(M)^{+}$consider a net $\left(x_{\alpha}\right) \subset M$ with $0 \leq x_{\alpha} \uparrow x^{\prime \prime}$ in $M^{\prime \prime}$. Hence, $x_{\alpha} \rightarrow x^{\prime \prime}$ for $\sigma\left(M^{\prime \prime}, M^{\prime}\right)$. Now, the rest of the proof is similar to that of Corollary $2.51 \Rightarrow 2$.

Conversely, assume that $H^{\prime \prime}(B(M)) \subset N$ and let $A \subset M$ be a set with $A \subset\left[-x^{\prime \prime}, x^{\prime \prime}\right]$ in $M^{\prime \prime}$ for some $0 \leq x^{\prime \prime} \in B(M)$. Since $\left(M^{\prime}, \beta\left(M^{\prime}, M\right)\right)$ is a Hausdorff locally convex-solid Riesz space, then by [2, Theorem 6.1] the order interval $\left[-x^{\prime \prime}, x^{\prime \prime}\right]$ of $M^{\prime \prime}$ is $\sigma\left(M^{\prime \prime}, M^{\prime}\right)$ compact. Now by similar arguments of [5, Proposition 2.11] it can be shown that $H(A)=$ $H^{\prime \prime}(A) \subset H^{\prime \prime}\left(\left[-x^{\prime \prime}, x^{\prime \prime}\right]\right) \subset N$ is relatively weakly compact as desired.

Remark 3.19. In the above theorem, we can replace the Dedekind completeness of $M$ with the topological completeness which is a more strong hypothesis; see [2, Theorem $3.24]$.

As a consequence, we get an improvement of the results on $b$-weakly compact operators [5, Propositions 2.8, 2.11] and [6, Proposition 1], which remain a part of the following corollary when specializing $M$ to be a Banach lattice.

Corollary 3.20. Let $M, N$ be a Fréchet lattice and a Banach space, respectively. Then, for a continuous operator $H: M \rightarrow N$ the following are equivalent:

(1) $H$ is a gbwc operator.

(2) $\left\|H x_{n}\right\| \rightarrow 0$, for every disjoint $b$-order bounded sequence $\left(x_{n}\right) \subset M^{+}$.

(3) $\left(H x_{n}\right)$ is convergent for every increasing b-order bounded sequence $\left(x_{n}\right) \subset M^{+}$.

(4) $\left(H x_{n}\right)$ is weakly convergent for every increasing b-order bounded sequence $\left(x_{n}\right) \subset$ $M^{+}$.

(5) $\left(H x_{n}\right)$ has a convergent subsequence for every increasing b-order bounded sequence $\left(x_{n}\right) \subset M^{+}$.

(6) $\left(H x_{n}\right)$ has a weakly convergent subsequence for every increasing b-order bounded sequence $\left(x_{n}\right) \subset M^{+}$.

(7) $H^{\prime \prime}\left(M^{\uparrow}\right) \subset N$.

If furthermore, $M$ has the Lebesgue property then we add

(8) $H^{\prime \prime}(B(M)) \subset N$.

\section{References}

[1] C.D. Aliprantis and K.C. Border, Infinite Dimensional Analysis, Berlin, Germany, Springer-Verlag, 2006. 
[2] C.D. Aliprantis and O. Burkinshaw, Locally Solid Riesz Spaces with Applications to Economics, Mathematical Surveys and Monographs 105, USA, Amer. Math. Soc., 2003.

[3] C.D. Aliprantis and O. Burkinshaw, Positive Operators, Dordrecht, The Netherlands, Springer, 2006.

[4] C.D. Aliprantis and R. Tourky, Cones and Duality, Providence, Rhode Island, USA, Amer. Math. Soc., 2007.

[5] S. Alpay, B. Altin and C. Tonyali, On Property (b) of Vector Lattices, Positivity, 7, 135-139, 2003. doi: 10.1023/A:1025840528211

[6] B. Altin, On b-Weakly Compact Operators on Banach Lattices, Taiwan. J. Math. 11 (1), 143-150, 2007.

[7] B. Altin and N. Machrafi, Some Characterizations of KB-operators on Banach Lattices and Ordered Banach Spaces, Turkish J. Math. 44, 1736-1743, 2020.

[8] P.G. Dodds, o-weakly compact mappings of Riesz spaces, Trans. Amer. Math. Soc. 214, 389-402, 1975.

[9] Z. Ercan, Interval-Bounded Operators and Order-Weakly Compact Operators of Riesz Spaces, Demonstr. Math. XXXI (4), 805-812, 1998.

[10] G. Köthe, Topological Vector Spaces I, Springer-Verlag New York Inc., 1969.

[11] P. Meyer-Nieberg, Banach Lattices, New York, USA, Springer, 1991. 